\title{
Percepções e práticas de agentes comunitários de saúde na atenção a usuários de drogas
}

\section{Perceptions and practices of community health workers in the care of drug users}

\author{
Karen Batista ${ }^{1}$, Bernardino Geraldo Alves Souto ${ }^{1}$
}

\section{RESUMO}

Introdução: No âmbito das políticas públicas vigentes no Brasil sobre atenção à saúde de pessoas que usam álcool e outras drogas, os agentes comunitários de saúde (ACS) da Estratégia de Saúde da Família (ESF) são profissionais de significativa importância para a busca ativa e para o cuidado dessas pessoas. No entanto, eles têm muita dificuldade em realizar tais ações, provavelmente devido à insuficiente qualificação técnica que recebem sobre abordagem adequada de usuários de drogas. Objetivo: Descrever as percepções e as expectativas de um grupo de ACS sobre usuários de álcool e outras drogas (UAOD), sobre as quais fundamentam suas respectivas práticas de cuidado. Métodos: Pesquisa clínico-qualitativa, aprovada por Comitê de Ética em Pesquisa com Seres Humanos, que entrevistou dez ACS experientes em cuidar de usuários de drogas. As entrevistas foram tratadas por estratégia ideográfica e organizadas nomoteticamente por conteúdos temáticos, os quais foram analisados sob os referenciais da clínica ampliada. Resultados: Os ACS querem cuidar adequadamente dos UAOD, mas não sabem como fazê-lo porque ainda convivem com noções leigas sobre essas pessoas e não recebem formação técnica suficiente. Conclusão: Esses ACS percebem a necessidade de se qualificarem e desejam essa qualificação para cuidar melhor de pessoas que usam álcool e drogas ilícitas. Portanto, para que as políticas públicas destinadas ao cuidado de quem tem problemas com o uso de drogas sejam eficazes, é indispensável a aplicação de estratégias de educação permanente capazes de transformar as práticas e as percepções desses profissionais.

Palavras-chave: agentes comunitários de saúde; saúde mental; usuários de drogas; Estratégia Saúde da Família.

\begin{abstract}
Introduction: In the context of public policies aimed at health care for people who use alcohol and illicit drugs in Brazil, the community health agents of the Family Health Strategy are very important for the active fetch and care of these people. However, they have difficulties to perform these actions, probably due to insufficient technical qualification as to appropriate approach of drug users. Objective: To describe the perceptions and expectations of a group of community health agents about drug users, on which they base their care practices. Methods: Clinical-qualitative research approved by the Research with Human Subjects Ethics Committee, which interviewed ten community health agents experienced the care of drug users. The interviews were treated by ideographic strategy and nomothetically organized by theme, later on being analyzed under the referential of extended clinics. Results: Community health agents want to take proper care of illicit drug users, but they do not know how to do it, probably because they still have lay beliefs about these people and do not receive enough technical training. Conclusion: These agents perceive their need to better qualify and wish to improve so they can take care of alcohol and other drugs' users. Therefore, in order for public policies aimed at the care of drug users to be effective, it is indispensable to apply permanent education strategies capable of transforming the practices and perceptions of these professionals.
\end{abstract}

Keywords: community health workers; mental health; drug users; Family Health Strategy. 


\section{INTRODUÇÃO}

A política do Ministério da Saúde para atenção integral à saúde de pessoas que usam drogas considera que o cuidado a tais sujeitos deve objetivar a reinserção social da pessoa e a redução de danos. Para isso, precisa acontecer no espaço de uma rede de serviços de saúde mental de base territorial e comunitária, integrada, articulada e resolutiva ${ }^{1,2}$.

Por outro lado, diretrizes internacionais preconizam que a atenção básica represente o eixo organizador da Rede de Atenção Psicossocial, garantindo cuidados primários em saúde mental e apoio comunitário ${ }^{3}$.

Nesse sentido, a Estratégia de Saúde da Família (ESF) torna-se um recurso importante para a política do Ministério da Saúde destinada ao cuidado de usuários de álcool e outras drogas (UAOD). O motivo é que essa estratégia se organiza sobre base territorial e comunitária e é capaz de atender às citadas diretrizes internacionais ${ }^{3}$.

No entanto, a literatura tem mostrado que, apesar de a ESF ser um modelo interessante ao cuidado de UAOD, os profissionais que nela atuam têm dificuldades em relação ao trabalho com essas pessoas. Essas dificuldades, por sua vez, determinam obstáculos ao alcance dos objetivos de reinserção social e de redução de danos, uma vez que limitam as práticas de cuidado a encaminhamentos e aconselhamentos superficiais derivados de: despreparo teórico e técnico das equipes; percepções estigmatizantes sobre usuários de álcool ou drogas ilícitas, com consequente tendência criminalizatória, segregatória e repressiva; desconhecimento das políticas públicas; e da ideia de institucionalização, de medicalização e de exclusão social dos sujeitos como estratégias de abordagem ${ }^{4-10}$.

Entre os profissionais da ESF com dificuldades em relação ao cuidado de UAOD é possível destacar o agente comunitário de saúde (ACS). Esse destaque justifica-se por ser este um profissional promotor da aculturação da equipe à comunidade e o articulador da integração entre ambas, e por ser quem busca ativamente as demandas locais por cuidado individual e coletivo. Como membro mais distal do sistema de saúde que entra em contato íntimo e direto com as pessoas na comunidade, o ACS é fundamental para o alcance dos objetivos da $\mathrm{ESF}^{4,11}$.

No entanto, a experiência mostra que são insuficientes as oportunidades de qualificação técnica oferecidas a ACSs para o conjunto de ações pelas quais são responsáveis.

Nesse espaço, ainda que a literatura já comente sobre as dificuldades de equipes de saúde da família em lidar com UOAD, pouco se ouviu até hoje, com olhar científico, o que os ACSs têm a dizer sobre tais dificuldades. Portanto, para ajudar a entender o problema exposto, desenvolveu-se o presente estudo com o objetivo de descrever e discutir as percepções, as práticas e as expectativas de um grupo de ACSs sobre usuários de drogas e sobre o respectivo processo de cuidado.

\section{MÉTODOS}

O presente artigo faz parte de uma pesquisa que foi submetida ao Comitê de Ética em Pesquisa da Universidade Federal de
São Carlos (UFSCar) por meio de seu envio online à Plataforma Brasil; a realização do trabalho foi aprovada pelo Parecer $\mathrm{n}^{\circ}$ 1.237.682, emitido em 21 de setembro de 2015. A partir daí, e com a autorização da gestão local do Sistema Único de Saúde (SUS), desenvolveu-se um estudo clínico-qualitativo ${ }^{12}$ por meio do qual entrevistaram-se, numa primeira etapa, 43 ACSs, em 9 unidades de saúde da família (USFs) de um município do interior do estado de São Paulo. Esses ACSs foram os que concordaram em participar como sujeitos da pesquisa por meio da assinatura de respectivo Termo de Consentimento Livre e Esclarecido (TCLE), conforme determina a Resolução n 466/12 do Conselho Nacional de Saúde ${ }^{13}$.

Inicialmente, foi criado um indicador especificamente para medir o grau de experiência (E) de cada ACS com o cuidado de UAOD, o qual teve a seguinte conotação matemática: $\mathrm{E}=\mathrm{TE} \times \mathrm{NU}$ (em que E=experiência no trabalho com UAOD; TE=dias acumulados de experiência nesse tipo de atividade; e NU=número de UAOD que o ACS já acompanhou ao longo de sua experiência profissional, incluindo os que acompanha atualmente).

Na Etapa 1, foram levantados 2 tipos de dados sobre os 43 participantes: aqueles que foram necessários para o cálculo do grau de experiência de cada ACS e dados sociodemográficos destinados à descrição amostral do conjunto dos participantes.

A finalidade do indicador do grau de experiência (E) de cada ACS foi ordenar esses sujeitos para serem entrevistados na segunda etapa, em uma sequência decrescente: a primeira entrevista foi feita com o ACS que obteve o maior valor para o indicador de experiência; a segunda com o que obteve o segundo maior valor; e assim sucessivamente. A intenção foi garantir a participação dos mais experientes no caso de não ser possível ou não ser necessário entrevistar todos, acreditando-se que, quanto mais experiente fosse o ACS, melhor seria o material produzido pela entrevista para os objetivos da pesquisa.

$\mathrm{Na}$ Etapa 2, os ACSs foram entrevistados um a um, em ordem decrescente a partir do que obteve o maior grau de experiência (E) até a saturação dos achados, o que aconteceu na décima entrevista. Portanto, a amostragem sobre a qual se produziram os resultados deste estudo foi por conglomerado institucional, fechada pelo critério da saturação e composta de dez entrevistados.

Nessa segunda etapa, realizou-se entrevista individual semiestruturada com o objetivo de levantar dados subjetivos sobre as práticas de cuidado e as percepções dos ACSs relacionadas ao uso e aos UAOD. Essas entrevistas foram gravadas em meio magnético e posteriormente transcritas para análise, a qual foi feita sobre blocos de conteúdos temáticos organizados por estratégia subsequentemente ideográfica e nomotética ${ }^{14}$.

Em termos práticos, o material subjetivo das entrevistas foi submetido a repetidas leituras flutuantes destinadas à apreensão e identificação, por parte dos pesquisadores, dos temas de interesse da pesquisa que foram abordados pelos entrevistados. Identificados esses temas, os mesmos foram categorizados segundo as ideias comuns que os caracterizavam como uma 
unidade temática (movimento ideográfico de identificação das ideias postas pelos entrevistados e de organização dessas ideias em categorias).

O passo seguinte foi o de localizar nas falas individuais os trechos que diziam respeito às categorias temáticas identificadas na fase ideográfica e organizá-los em conjuntos, de acordo com cada uma dessas categorias (movimento nomotético), criando-se um corpo de achados ${ }^{14}$.

Os conjuntos, assim produzidos e organizados, tiveram cada fala analisada individualmente à luz do referencial da integralidade do cuidado e da clínica ampliada e compartilhada ${ }^{15,16}$. Somadas dentro do conjunto (dentro da categoria temática), a contribuição de cada entrevistado sobre o respectivo tema específico oportunizou uma compreensão particular ampliada de cada fenômeno analisado segundo diferentes olhares pessoais dentro da mesma categoria, em cada categoria estudada.

Quanto aos referenciais da integralidade do cuidado e da clínica ampliada e compartilhada sobre os quais se basearam as interpretações das falas dos sujeitos, os mesmos partem do princípio de que cada pessoa é um todo integrado ao seu meio social e ecológico de modo a reconhecer os sentimentos, as percepções, as reações, as atitudes, os sinais e os sintomas dos indivíduos como contextualizados em suas dimensões sociais e clínicas, e em seus projetos existenciais $^{15,16}$.

Em síntese, trata-se de um estudo qualitativo que utilizou o método clínico da escuta para a realização de entrevistas semiestruturadas com dez ACSs amostrados por conglomerado institucional fechado por saturação. Esses ACSs foram pré-selecionados entre os mais experientes em cuidar de pessoas que usam álcool e outras drogas dentro do universo amostral disponível e responderam sobre suas práticas e percepções a respeito dessas pessoas e do cuidado que prestam às mesmas.

As entrevistas foram analisadas segundo o conteúdo temático identificado e organizado por meio de uma construção ideográfica e nomotética, à luz dos referenciais da integralidade do cuidado e da clínica ampliada e compartilhada ${ }^{12,15,16}$.

\section{RESULTADOS E DISCUSSÃO}

Aceitaram participar da pesquisa 43 ACSs, mediante TCLE. Entretanto, no décimo entrevistado mais experiente em cuidar de UAODs houve saturação dos achados, dispensando-se a necessidade de se entrevistarem os demais 33 voluntários.

Um aspecto que chamou a atenção foi o fato de haver, entre os entrevistados, alguns que tinham participado do curso "Caminhos do cuidado: formação em saúde mental (crack, álcool e outras drogas)", oferecido pelo Ministério da Saúde com a intenção de qualificar ACSs para a assistência a pessoas que necessitam de atenção devido ao fato de usarem álcool ou outras drogas. Essa ocorrência foi aleatória; portanto, não intencional no processo de amostragem, mas mera coincidência desproposital. Não obstante, tal fato oportunizou ampliar a compreensão dos achados ao se considerar esse aspecto específico na análise dos dados conforme se verá adiante, ao longo da discussão.

O Quadro 1 discrimina as variáveis sociodemográficas e o indicador de experiência dos dez ACSs que responderam à entrevista subjetiva semiestruturada durante a segunda etapa do estudo.

Quanto às categorias temáticas identificadas, observou-se que os ACSs trataram dos seguintes assuntos ao longo das entrevistas: acolhimento, modelo assistencial biomédico, limitação da autonomia profissional, influência cultural leiga sobre o cuidado, possibilidades oferecidas pela reflexão crítica, estigma relacionado ao uso de drogas e política pública frente ao conflito entre a ciência e a cultura. Os achados respectivos a cada uma dessas categorias expõem-se a seguir.

\section{Acolhimento}

A princípio, observou-se tentativa dos ACSs em acolher o UAOD:

[...] eu acho que orientar [...] mostrar que você se importa com ele. Não só por conta daquele problema da droga mas assim, de um modo geral. [...] como que ele tá, se ele tá estudando, se ele tá trabalhando, como é que tão as coisas em casa, né (ACS 4).

Quadro 1: Indicador de experiência, dados sociodemográficos e participação no curso Caminhos do Cuidado dos 10 Agentes Comunitários de Saúde que responderam à entrevista qualitativa semiestruturada

\begin{tabular}{|c|c|c|c|c|c|}
\hline ACS & $\begin{array}{l}\text { Valor obtido no indicador } \\
\text { de experiência (E) }\end{array}$ & $\begin{array}{l}\text { Idade em } \\
\text { anos }\end{array}$ & Escolaridade & $\begin{array}{l}\text { Morador da } \\
\text { comunidade }\end{array}$ & $\begin{array}{c}\text { Frequentou o curso } \\
\text { "Caminhos do cuidado"* }\end{array}$ \\
\hline 1 & 289.200 & 53 & Ensino médio completo & Não & Sim \\
\hline 2 & 255.500 & 31 & Ensino médio completo & Não & Não \\
\hline 3 & 109.500 & 32 & Ensino superior completo & Não & Não \\
\hline 4 & 32.120 & 53 & Ensino superior incompleto & Não & Sim \\
\hline 5 & 26.565 & 58 & Ensino superior completo & Não & Sim \\
\hline 6 & 22.500 & 42 & Ensino superior incompleto & Sim & Não \\
\hline 7 & 16.425 & 35 & Ensino superior incompleto & Sim & Sim \\
\hline 8 & 9.700 & 33 & Ensino médio completo & Não & Sim \\
\hline 9 & 3.880 & 25 & Ensino médio completo & Sim & Sim \\
\hline 10 & 960 & 44 & Ensino médio completo & Não & Sim \\
\hline
\end{tabular}

ACS: agente comunitário de saúde; *curso de capacitação de agentes comunitários de saúde em atenção a usuários de álcool e outras drogas na atenção básica editado pelo Ministério da Saúde no ano de 2013 e realizado no município de Rio Claro em 2015. 
O acolhimento é um modo de cuidar em saúde que implica em compreender a pessoa em suas demandas físicas, sociais e psíquicas, de modo que suas necessidades possam ser reconhecidas e trabalhadas de maneira articulada e integral ${ }^{17}$.

Para isso propõe-se, entre outras práticas, ações transdisciplinares caracterizadas pela horizontalização das relações entre os provedores do cuidado e a pessoa com necessidades de atenção, as quais podem ter o ACS como esse provedor ${ }^{16,18}$.

\section{Modelo assistencial biomédico}

No processo em que se propõe ofertar um cuidado integral, incluiu-se a abordagem médica biológica, entendendo-se que o uso de álcool e outras drogas está associado também a problemas orgânicos:

[...] vamos tentar ver se ele consegue vim no médico, pro médico pedir uns exames nele, porque faz muuuuito tempo que ele não vem no médico, a gente não sabe como ele tá (ACS 6).

No entanto, a expectativa de intervenção médico-biológica no processo de cuidado de uma pessoa que usa álcool e outras drogas, a exemplo do exposto pelo entrevistado acima, pode ser consequência de uma percepção de que tal uso representa uma doença crônica adquirida ${ }^{19}$. Nesse caso, álcool e outras drogas podem ser metaforizados como os agentes etiológicos e seu usuário como o hospedeiro definitivo desse agente que não pode ser eliminado do organismo. Uma vez percebido como problema de ordem biopatológica, pode ser que a condição de UAOD seja vista como algo que demanda uma intervenção diagnóstica e uma prescrição médica.

Nesse sentido, a premissa de que, obrigatoriamente, tem que haver um diagnóstico e um tratamento para tudo o que é percebido como desvio funcional tende a fazer com que o cuidado seja visto como essencialmente dependente do médico ou de tecnologias duras ${ }^{20}$ :

Eu acho que o governo deveria dar mais clínicas, ter uma clínica específica para drogados, sem limite [...] (ACS 5).

[...] no pior o que eu imagino no paciente preso ou morto. Dependendo do grau da droga, dependendo do uso que ele faz, ou também ele pode ser internado numa clínica e se recupe$\operatorname{rar}(\mathrm{ACS} 1)$

Nesse caso, a institucionalização do UAOD, seja no sistema prisional ou em regime de internação hospitalar, também é vista como uma estratégia intersetorial contra o uso de álcool e outras drogas. Entretanto, em inspeções realizadas em instituições de internação de usuários de drogas, observaram-se dificuldades como superlotação, insuficiência de recursos humanos, precariedade das condições físicas, ausência de projeto terapêutico singular, elevado número de pessoas aguardando exames, intervenções semelhantes para pessoas com problemas diferentes, denúncias de agressões e de maus tratos e o uso de estratégias segregatórias e punitivas ${ }^{21,22}$.

\section{Limitação da autonomia profissional}

Se um ACS desenvolver uma percepção de que é fundamental a institucionalização do UAOD, poderá se sentir tecnicamente limitado para contribuir para o cuidado demandado e, com isso, ter obnubilada a noção da abordagem integral em que seu apoio é fundamental:

[...] não tem como você falar muita coisa [...] a gente tenta orientar a pessoa a vim aqui. Mas uma prática de saúde que a gente não pode entrar na questão social (ACS 3).

No espaço em que surge essa percepção de limitação técnica que compromete a integralidade do cuidado, observou-se uma tendência dos ACSs de encaminharem o usuário de drogas para tratamento; porém, sob o entendimento de que a pessoa não desejava o cuidado oferecido, fundamentado na percepção de que há desinteresse da própria pessoa em relação ao projeto terapêutico proposto:

[...] aí a gente tentou encaminhar ele para alguns... encaminhou ele pro CAPS, a mãe também, [...] mas assim ele aparentemente ele não quer sair dessa vida pelo jeito [...] (ACS 6).

A gente tem que tomar o máximo de cuidado né. Tem pessoas que elas não querem ser ajudadas (ACS 3).

Nesse caso, parece que a compreensão do ACS foi de que a pessoa que usa álcool e outras drogas resiste ao projeto terapêutico sugerido.

\section{Influência cultural leiga sobre o cuidado}

Também se observaram práticas de cuidado de orientação leiga e paternalista, possivelmente fundamentadas num sentimento de compaixão do ACS pelo UAOD, capaz de resultar numa caridade de base religiosa:

Oriento a buscar tratamento, eu oriento a ir pra uma igreja, eu dou suporte e encaminhamento pra vários seguimentos pra que ele melhore (ACS 5).

[...] eu acho que em qualquer situação você tem que ter paciência, amor né. Deus sobre todas as coisas e ir com calma [...] (ACS 4).

A percepção da caridade com apelo religioso como recurso assistencial, quando descontextualizado dos aspectos científicos demandados pela condição de saúde da pessoa assistida, pode concorrer com a necessidade de provisão de um cuidado 
tecnicamente adequado e até mesmo desviar a assistência prestada da que é indicada pelas políticas públicas e pela evidência científica ${ }^{19}$. Não é raro, ainda, que esse apelo derive da percepção de exaustão da possibilidade terapêutica:

[...] ele chegou no fundo do poço e ele pediu mesmo para Deus [...] Aí Deus na hora que deu o sinal para ele [...] ele conseguiu mudar (ACS 6).

No caso de o cuidado subordinar-se inteiramente à religiosidade, isso poderá ser tanto causa como consequência de uma subtração na autonomia da pessoa com necessidades de saúde, sustentada em modelos doutrinários da moral que defendem o controle sobre o sujeito como estratégia para a sua reabilitação. Nesse caso, o que se entende por reabilitação também está subordinado ao olhar de quem impõe o controle.

O ajuizamento moral sobre o uso e o UAOD apareceu na fala de um ACS no contexto da sua dificuldade em admitir a possibilidade de a pessoa sob seus cuidados ser usuária, como se essa revelação representasse algo devastador:

[...] Aí comecei a conversar com ele e chegou na parte:

- Usa drogas? É, e a gente já vai no "Não" [...] Nunca imaginei que ele fosse usar... Aí eu (perguntei):

- O senhor usa? Não... eu vou ser sincero pra você, não pode mentir, não pode seu João, fala a verdade!

- Não, eu uso, eu fumo maconha [...] (ACS 6).

Devastador porque viola a expectativa estereotípica que se tem sobre uma pessoa que usa droga:

- Mas o senhor fuma mais alguma...?

- Não, só a maconha mesmo.

Mas é uma pessoa de boa, de bem com a vida assim sabe, não é uma coisa que eu acho que ele é assim, viciado mesmo, sabe, e ele é uma pessoa bem bacana, assim, ele cata reciclagem assim na rua, eu nunca ouvi falar que ele maltratou ninguém, é uma pessoa educada, sabe? Ah mas foi muito engraçado esse dia assim sabe... (risos). [...] Assim nenhuma complicação para família, não tá destruindo a família, tá tudo mundo assim, sabe, tudo tranquilo [...] (ACS 6).

\section{Possibilidades oferecidas pela reflexão crítica}

Não obstante, essa experiência de contato com UAOD, sem que isso repercuta negativamente no ambiente ou no comportamento ético-social sob responsabilidade do usuário, pode facilitar ao ACS uma reflexão produtiva em favor de uma melhor compreensão e tolerância em relação a esse usuário:

[...] eu fui conversar com ele sobre ele usar droga tal [...] Ele falou: "eu não prejudico ninguém, eu trabalho, eu tenho meu emprego, eu cuido da minha filhinha, pago minhas contas, ah qual que é o problema?" Eu sinceramente não tenho nada contra a pessoa que usa maconha (ACS 10).

No âmbito dessa tolerância pode surgir um desejo de conciliação com o UAOD no sentido de aceitá-lo desde que o mesmo não faça uso nocivo da droga:

[...] Bom, seria bom se as pessoas não usassem droga mas como hoje isso não é possível, tentar ficar mais junto da pessoa, conversar, pra eles num vê a gente como inimigo. [...] A gente tem preconceito, às vezes tem até medo né, sair à noite por causa dessas pessoas. Mas é bom que unificasse tudo [...] (ACS 10).

Contudo, houve quem fosse ainda mais tolerante e propusesse uma abordagem solidária e menos condicional, fundamentada numa reflexão crítica social:

[...] Todo mundo tem na família ou conhecido que usa. Então vamo tentar ajudar. Mas vamo ser amigo, vamo ser normal, sei lá. Não deixar a pessoa se sentir excluída (ACS 10).

Ninguém é normal, assim, basta um entender o outro [...] você é aceito se você é normal, se você faz umas coisas meio fora do padrão você não é normal não (ACS 10).

\section{O estigma relacionado ao uso de drogas}

Porém, o que predominou foi a percepção de que, invariavelmente, há algo degenerado e ameaçador no UAOD. E quando não é assim, foge ao conceito estabelecido ao ponto de surpreender e soar ilógico, inacreditável e fora dos padrões. A maior parte dos entrevistados deixou transparecer uma percepção de que, se a pessoa usa droga, necessariamente tem algo de ruim a oferecer no contexto de suas relações e, portanto, representa uma condição que não deveria existir. Se ela não tem isso de ruim é porque, na verdade, não é o estereótipo de usuária de droga que está no imaginário coletivo. Ou seja, há um preconceito depreciativo, talvez estigmatizante, em relação a quem usa droga, mesmo por parte de ACSs cuja primeira função é acolher essa pessoa, demonstrando uma dificuldade desse profissional em compreender que o uso de droga pode fazer parte da constituição existencial do indivíduo sem que isso implique em problemas ${ }^{23}$ :

Preconceito a gente tem [...]. No fundo, no fundo, todo mundo tem preconceito [...] (ACS 10).

[...] ela não é vista como uma pessoa, um ser, ela é vista como uma coisa que dá trabalho, uma sem sentido. Ela é uma pessoa que dificilmente alguém dá a mão. Todo mundo fala, todo mundo evita, porque o preconceito existe contra a droga, contra qualquer outro tipo de dependência. Existe preconceito, e a pessoa sofre muito e a família, os amigos, todas as pessoas que cercam ela vão sofrer junto (ACS 5). 
E aí o estigma aparece claramente na fala a seguir:

Ah um receio né. Cria um receio, uma barreira. Não vou explicar assim um nome pra isso [...] Até onde né, a pessoa que tá usando a droga já tá num certo grau de uso, ela pode me atingir? Com o contato mais direto (ACS 3).

Nesse caso, surge o óbvio efeito do estigma que, segundo Goffman ${ }^{23}$, desqualifica o estigmatizado:

[...] eles sentem vergonha. Eu acho que eles sentem vergonha. É o meu ponto de vista [...] Da própria fraqueza deles. De algum ponto que eles não conseguem trabalhar (ACS 1).

Ainda segundo as ideias de Goffman ${ }^{23}$, sendo o estigma uma marca que se impinge a uma pessoa com o fim de identificá-la com o indesejável, essa marca interfere, pois, na relação de quem a percebe com aquele que se tornou maculado no sentido de despertar um desejo pela segregação do estigmatizado. Esse fenômeno, na percepção de um ACS, pode resultar numa ação excludente do UAOD, capaz de obstruir o acesso dessa pessoa a cuidados adequados de saúde ${ }^{24}$.

Nesse ambiente de significados, pode tornar-se patente, na visão de ACSs, a associação entre uso de álcool e outras drogas a um fenômeno que é degenerado a partir de sua própria natureza:

[...] Eu acho que assim, é bem [...] uma fraqueza de espírito. Mas eu acho que é a emoção, um distúrbio emocional da pessoa [...] (ACS 7).

Porque as pessoas hoje não, não têm [...] aquela família unida, que dá mais estrutura, mais base sabe pra um futuro melhor (ACS 5)

Não sei...tem tanta gente boa que usa...gente mal você vai falar que é, que é a situação dela. Mas e o cara legal, o cara que estuda, o cara que faz faculdade...sei lá. Por que que usa droga? [...] (ACS 10).

\section{A política pública frente ao conflito entre a ciência e a cultura}

Por outro lado, os ACSs demonstraram vivenciar um conflito entre suas percepções culturais relacionadas aos UAOD e o discurso científico ou as políticas públicas a que tiveram acesso.

Nesse sentido, destacou-se o curso "Caminhos do cuidado", ofertado nacionalmente pelo Ministério da Saúde para a qualificação de profissionais da atenção básica sobre cuidados de $\mathrm{UAOD}^{17}$ :

[...] você tem que parar e pensar naquilo que você aprendeu porque pra gente, por mais que você conviva, você nunca teve uma...eu pelo menos nunca tive uma orientação que nem agora a gente teve no Caminhos, né... Caminhos do Cuidado, então você fica lembrando, mas nossa, mas eu aprendi que é assim, assim, assim. Então eu procuro respirar e procuro colocar em prática aquilo que a gente aprendeu (ACS 4).

Essa questão da formação profissional de ACSs apresentou-se como importante possibilidade para a aplicação das políticas públicas sobre cuidados de pessoas que usam álcool e drogas ilícitas. Como dito, há um conflito entre a influência da cultura de fundamentação leiga e a ciência na percepção de ACSs sobre essas pessoas e o fenômeno que as envolve. Esse conflito prejudica a integralidade do cuidado por dificultar a participação qualificada dos ACSs na assistência. Entretanto, a formação técnica mostra-se potencialmente capaz de influenciar a atuação dos mesmos em favor do que se pretende em termos de atenção aos UAOD:

Mas eu acho que a gente pode contribuir bastante pra que tenha um bom desfecho né. Aquela parte que a gente estudou sobre redução de danos, eu acho que se a gente conseguir reduzir um pouquinho que seja [...] eu faço a minha parte [...] (ACS 4).

Interessante foi que os ACSs se mostraram sensíveis a esse progresso:

Eu acho que a gente tinha que ir buscar mais, mas a gente tem que ser preparado pra lidar com isso também [...] (ACS 2).

Percebe-se que os próprios ACSs refletiram criticamente sobre sua potência profissional e sobre a necessidade de qualificarem a atenção que oferecem aos UAOD. No entanto, para que tal qualificação ocorra, é fundamental que esses profissionais sejam formados para isso e que recebam apoio para desenvolver as respectivas ações de saúde no território.

A literatura, as políticas públicas e as diretrizes internacionais reconhecem que a ESF é uma ferramenta essencial para a provisão do cuidado qualificado aos UAOD, que tem como objetivo a reinserção social do sujeito e a redução de danos. Dentro dessa estratégia, destacou-se o papel dos ACSs.

Nesse foco, respeitadas as limitações inerentes à metodologia utilizada neste estudo e o universo investigado, estabelecidas pela subjetividade dos achados, pela característica específica dos sujeitos e pela organização do ambiente assistencial em que trabalham, observou-se que os ACSs têm tendência acolhedora sobre os UAOD na tentativa de ofertarem uma atenção ampliada e integral. Contudo, essa tentativa mostrou-se prejudicada pela falta de oportunidade de qualificação técnica; pela influência de percepções de ordem sociocultural estigmatizantes sobre os UAOD e por um sentimento de baixa autoeficácia profissional, o qual sustenta uma crença equivocada de que os modelos assistenciais hospitalares, médico-centrados ou restritivos da autonomia dos sujeitos são mais eficazes.

Esse conjunto de obstrutores, aliado a uma falta de adequada estrutura de suporte assistencial secundário e terciário, dificulta a 
aplicação correta das políticas públicas vigentes para o cuidado de UAOD por parte das equipes de saúde da família, dando a impressão de uma ineficiência sistêmica das próprias políticas públicas.

Não obstante, os ACSs compreendem suas limitações técnicas e identificam a importância de qualificação para o cuidado de UAOD, entendendo que é possível prover uma atenção melhor do que aquela que é ofertada. Apontaram, inclusive, que muitas de suas ações leigas destinadas ao cuidado de UAOD, as quais resultam numa abordagem pouco qualificada e ineficiente em relação ao objetivo das políticas públicas, derivam da tentativa de cuidarem mesmo na falta de recursos pessoais e profissionais para isso. Entretanto, como dito, mostraram-se interessados em fazer melhor e, portanto, reivindicam oportunidade de conquista desses recursos.

É fundamental garantir espaços regulares de educação permanente para que os ACSs e demais profissionais da ESF tenham condições de refletir sobre as políticas de saúde mental, bem como problematizar e construir modelos de atenção à saúde numa perspectiva ampliada e emancipatória sobre os usuários de drogas.

Em síntese, os ACSs demonstraram interesse por cuidar de UAOD, mas não sabem como fazê-lo adequadamente. Essa condição os deixa numa situação de insegurança pessoal e profissional frente a esses sujeitos e às suas próprias limitações, o que leva ao desperdício da potencialidade que têm.

Portanto, sugere-se incluir entre as políticas públicas a aplicação de estratégias pedagógicas de qualidade e intensidade suficientes para tornar o ACS um profissional capaz de exercer uma clínica ampliada e compartilhada no âmbito da ESF, bem como a produção de novos estudos sobre o tema por meio de estratégias metodológicas que permitam avaliar a influência dos treinamentos nas percepções e nas práticas do ACS.

\section{REFERÊNCIAS}

1. Brasil. Ministério da Saúde. Secretaria Executiva Coordenação Nacional de DST e AIDS. A Política do Ministério da Saúde para atenção integral a usuários de álcool e outras drogas. Brasília: Ministério da Saúde; 2003.

2. Brasil. Conselho Nacional de Secretários de Saúde. Portaria n. 3.088, de 23 de dezembro de 2011. Institui a Rede de Atenção Psicossocial no SUS. Diário Oficial da União. Brasília; 2011.

3. Dimenstein M, Lima Al, Macedo JP. Integralidade em saúde mental: coordenação e continuidade de cuidados na Atenção Primária. In: Paulon S, Neves R. Saúde Mental na Atenção Básica: a territorialização do cuidado. Porto Alegre: Sulina; 2013. p.39-60.

4. Araújo LF, Castanha AR, Barros APR, Castanha CR. Estudo das representações sociais da maconha entre agentes comunitários de saúde. Ciênc Saúde Coletiva. 2006;11(3):827-36. http://dx.doi.org/10.1590/S1413-81232006000300030

5. Fernandes A, Stein AT, Giugliani C. Conhecimentos dos agentes comunitários de saúde sobre crack e outras drogas. Rev Bras Med Fam Comunidade. 2015;10(34):1-10. http://dx.doi.org/10.5712/rbmfc10(34)924

6. Habimorad PHL, Martins STF. O abuso e dependência de drogas no território: desafios e limites dos Agentes Comunitários de Saúde de uma Unidade de Saúde da Família. Pesq Práticas Psicossociais. 2013;8(1):63-74.

7. Neves R, Paulon S, Nardi HC, Tschiedel R, Zambenedetti G, Piccinini CA, et al. Rastreando o cuidado em saúde mental pela mão dos Agentes Comunitários de Saúde: um percurso de pesquisa. In: Paulon S, Neves R. Saúde Mental na Atenção Básica: a territorialização do cuidado. Porto Alegre: Sulina; 2013. p.113-48.

8. Queiroz IS, Gomes AP, Reis AL, Knupp DFD, Aquino CR. Repertórios interpretativos de profissionais da rede de saúde mental e atenção primária de Belo Horizonte sobre uso de drogas. Saúde Debate. 2014;38(100):80-93. http://dx.doi.org/10.5935/0103-104.20140005
9. Silva RAN, Zambenedetti G, Piccinini CA. O trabalho dos Agentes Comunitários de Saúde no cuidado com pessoas que usam drogas: um diálogo com a PNH. Rev Polis Psique. 2012;2(3):59-72.

10. Silveira PS, Martins LF, Ronzani TM. Moralização sobre o uso de álcool entre agentes comunitários de saúde. Psicol Teor Prática. 2009;11(1):62-75

11. Brasil. Ministério da Saúde. Secretaria de Atenção à Saúde. Departamento de Atenção Básica. Política Nacional de Atenção Básica. Brasília: Ministério da Saúde; 2012.

12. Turato ER. Tratado da metodologia da pesquisa clinico qualitativa: construção teórico-epistemológica, discussão comparada e aplicação nas áreas da saúde e humanas. $2^{\mathrm{a}}$ ed. Petrópolis: Vozes; 2003.

13. Brasil. Conselho Nacional de Saúde. Resolução $n^{\circ} 466$, de 12 de dezembro de 2012. Diário Oficial da União. Brasília; 2012.

14. Lucena AF. Análise ideográfica e nomotética na pesquisa qualitativa com abordagem fenomenológica. Disponível em: http://www.lume.ufrgs.br/handle/10183/12739. Acesso em: 22 jul. 2017.

15. Campos GWS. A clínica do sujeito: por uma clínica reformulada e ampliada. In: Campos GWS. Saúde Paidéia. São Paulo: Hucitec: 2003. p.51-67.

16. Mattos RA. Os sentidos da integralidade: algumas reflexões acerca de valores que merecem ser defendidos. In: Pinheiro $R$, Mattos RA. Os sentidos da integralidade na atenção e no cuidado à saúde. $8^{\mathrm{a}}$ ed. Rio de Janeiro: Cepesc IMS/UERJ Abrasco; 2009. p.43-68.

17. Brasil. Ministério da Saúde. Secretaria de Gestão do Trabalho e da Educação na Saúde Departamento de Gestão da Educação na Saúde. Projeto "Caminhos do Cuidado" Formação em saúde mental (crack, álcool e outras drogas) para agentes comunitários de saúde e auxiliares/técnicos em enfermagem da Atenção Básica. Brasília: Ministério da Saúde; 2013. 
18. Schneider DR. Horizonte de racionalidade acerca da dependência de drogas nos serviços de saúde: implicações para o tratamento. Ciênc Saúde Coletiva. 2010;15(3):687-98. http://dx.doi.org/10.1590/S1413-81232010000300011

19. Alarcon S, Belmonte PR, Jorge MAS. O campo de atenção ao dependente químico. In: Alarcon S, Jorge MAS. Álcool e outras drogas: diálogos sobre um mau-estar contemporâneo. Rio de Janeiro: Fiocruz; 2012. p.63-82.

20. Conselho Federal de Psicologia. Relatório da $4^{a}$ Inspeção Nacional de Direitos Humanos: locais de internação para usuários de drogas. $2^{a}$ ed. Brasília: Comissão Nacional de Direitos Humanos; 2011.

21. Conselho Regional de Psicologia de São Paulo. Dossiê: Relatório de inspeção de comunidades terapêuticas para usuárias(os) de drogas no estado de São Paulo. Mapeamento das violações de direitos humanos. Campanha Estadual de Direitos Humanos. Violência do Estado ontem e hoje, da exclusão ao extermínio. São Paulo: Conselho Regional de Psicologia de São Paulo; 2016

22. Alarcon S. A Síndrome de Elêusis: considerações sobre as políticas públicas no campo de atenção ao usuário de drogas. In: Alarcon S, Jorge MAS. Álcool e outras drogas: diálogos sobre um mau-estar contemporâneo. Rio de Janeiro: Fiocruz; 2012 p. $45-62$

23. Goffman E. Estigma: notas sobre a manipulação da identidade deteriorada. $4^{a}$ ed. Rio de Janeiro: Livros Técnicos e Científicos; 1988.

24. Ronzani TM, Noto AR, Silveira PS. Reduzindo o estigma entre usuários de drogas: guia para profissionais e gestores. Juiz de Fora: Editora da UFJF; 2015 